\title{
Estado, educación y pueblos indígenas en los Andes ecuatorianos
}

\section{State, education and indigenous peoples in the Ecuadorian Andes}

Investigación

\author{
Sebastián Granda Merchán \\ Universidad Politécnica Salesiana - Ecuador \\ sgranda@ups.edu.ec
}

Recibido: 27 de junio de 2016 / Aceptado: 19 de octubre de 2016

\section{Resumen}

Este artículo presenta algunos de los resultados de la investigación desarrollada por el autor en el marco de su tesis doctoral. El artículo analiza el impacto que el proceso de institucionalización de la educación intercultural bilingüe en el Ecuador tuvo en las iniciativas de educación indígena de carácter comunitario. El trabajo de investigación se fundamentó en los aportes de la Pedagogía Crítica y de la Antropología Latinoamericana: de la primera tomó el concepto de educación como práctica cultural y política, y de la segunda recuperó la perspectiva analítica de la teoría de control cultural. En términos metodológicos, la investigación optó por realizar el estudio de caso de una experiencia de educación indígena emblemática del país: el Sistema de Escuelas Indígenas de Cotopaxi (SEIC), y para la recolección de información se trabajó a partir de la historia oral y la revisión de fuentes primarias. La hipótesis que se busca argumentar es que el proceso de institucionalización de la educación intercultural bilingüe terminó minando el carácter comunitario del SEIC y estandarizando su propuesta formativa.

Palabras clave: Estado, educación indígena, pueblos indígenas, Andes, Ecuador

\section{Summary}

This article presents some of the results of the doctoral thesis developed by the author. The article analyzes the impact that the process of institutionalization of intercultural bilingual education in Ecuador had in those initiatives of education controlled by indigenous communities. The research was supported on the contributions of Critical Pedagogy and Latin American Anthropology: from the first one, it took the concept of education as a cultural and politic practice; from the second one, it took the analytical perspective of the cultural control theory. Related to the methodology, the research made a study case of an emblematic experience of indigenous education in the country: the Sistema de Escuelas Indígenas de Cotopaxi (SEIC), and data was collected through oral history and review for primary sources. The hypothesis that the article seeks to argue is that the process of institutionalization of the intercultural bilingual education finished undermining the community nature of the SEIC and standardizing its educational proposal.

Keywords: State, indigenous education, indigenous people, Andes, Ecuador

Forma sugerida de citar: Granda, Sebastián (2016). Estado, educación y pueblos indígenas en los Andes ecuatorianos. Alteridad, 11(2), pp. 221-230. 


\section{Introducción}

En 1988, fruto de la presión y la negociación del movimiento indígena con el Gobierno de la Izquierda Democrática, se concertó el proceso de institucionalización de la educación intercultural bilingüe (EIB) en el Ecuador. Dicho proceso supuso la construcción de una jurisdicción propia para la educación indígena, así como la creación de una instancia al interior del Ministerio de Educación para su gestión y administración: la Dirección Nacional de Educación Intercultural Bilingüe (DINEIB), con sus respectivos brazos operativos en el nivel local: las direcciones provinciales.

Con el proceso de institucionalización de la EIB, todas las iniciativas de educación indígena que venían operando en el país quedaron adscritas a la jurisdicción de EIB y pasaron a depender de la DINEIB. Entre dichas iniciativas constaban aquellas impulsadas por el Estado, la cooperación internacional y la iglesia; pero también aquellas iniciativas gestadas y sostenidas por las mismas organizaciones y comunidades indígenas.

La presente investigación nació de la preocupación por el significado del proceso de institucionalización de la educación indígena en el país y el impacto que dicho proceso tuvo en las iniciativas de educación indígena gestadas y controladas por las comunidades indígenas. La pregunta que orientó la investigación fue la siguiente: ¿cuáles fueron los cambios que las iniciativas de carácter comunitario experimentaron el momento en que pasaron a formar parte del Estado, y se sujetaron a sus políticas, regulaciones y tiempos?

La hipótesis que se busca argumentar en el texto que sigue es que el proceso de institucionalización tuvo un impacto contradictorio en las iniciativas de educación indígena comunitarias, pues a la par que contribuyó a asegurar la sostenibilidad de aquellas en el tiempo, terminó resquebrajando su carácter comunitario y desmantelando sus propuestas de formación.

El artículo se encuentra organizado en tres partes: en la primera se describe el enfoque teórico que fundamentó el trabajo de investigación y la metodología utilizada. En la segunda parte se analiza las características centrales del SEIC y los principales cambios que vivió la experiencia educativa por efecto de su institucionalización. Y, en la última, se plantean las conclusiones y se discuten las condiciones de posibilidad para el desarrollo de iniciativas de educación que, a la par que formen parte del Estado, sean sensibles con la realidad de las comunidades indígenas y respondan a sus proyectos como colectividades.

\section{Fundamentación teórica y metodológica}

En relación a las herramientas teóricas utilizadas, la investigación recuperó algunos de los aportes de la Pedagogía Crítica y de la Antropología Latinoamericana. De la Pedagogía Crítica se tomó el concepto de educación como práctica cultural y política. Desde esta perspectiva, la educación deja de ser entendida como un asunto meramente técnico, que se reduce a aspectos que tienen que ver exclusivamente con la didáctica; y pasa a ser concebida como una práctica que está condicionada por el contexto social y político, y que está directamente inmiscuida en la producción y regulación de discursos, significados y subjetividades (Giroux, 1995).

Lo anterior explica por qué la educación constituye un terreno de lucha fundamental, pues todo intento por reproducir el orden social o transformarlo pasa, necesariamente, por la puesta en juego de una estrategia y una política educativa. Explica, también, por qué la educación ha sido un bien valorado por aquellos sectores que no han tenido acceso a ella, como ha sido el caso, por ejemplo, de amplios sectores de la población indígena del Ecuador y de la Región Andina, que han visto en la educación una herramienta fundamental para su proceso organizativo y de lucha, pero también para su afirmación cultural (Walsh, 2013).

De la Antropología Latinoamericana se recuperó la propuesta de la teoría del control cultural de Guillermo Bonfil Batalla (1991), y, de manera particular, su perspectiva de análisis de las relaciones 
que se desarrollan entre grupos étnicos que viven en condiciones de asimetría. La teoría del control cultural constituye un esfuerzo importante por comprender las persistencias y cambios culturales de la población indígena a partir de la categoría de control cultural, entendida esta última como la capacidad social que tienen los grupos étnicos para controlar los elementos culturales y usarlos para un fin determinado (Pérez, 2013).

En términos sintéticos, la teoría del control cultural plantea que todo grupo étnico es portador de cuatro ámbitos de cultura, que varían dependiendo de la pertenencia de los elementos culturales pero también de la capacidad de decisión que el grupo tiene sobre ellos: el ámbito de cultura autónoma (elementos culturales propios sobre los cuales el grupo tiene capacidad de decisión), el ámbito de cultura enajenada (elementos propios sobre los cuales el grupo no tiene ninguna capacidad de decisión), el ámbito de cultura apropiada (elementos ajenos sobre los cuales el grupo ha pasado a tener decisión), y el ámbito de cultura impuesta (elementos ajenos sobre los cuales el grupo no tiene decisión) (Bonfil, 1991).

Plantea, también, que en el contexto de las relaciones interétnicas en condiciones de asimetría, dichos ámbitos cambian continuamente por efecto de los procesos que se ponen en juego tanto desde el lado del grupo dominante como desde el grupo dominado. Desde de los grupos dominantes, los tres principales procesos que se ponen en práctica son la imposición, la supresión y la enajenación. Desde el lado de los grupos dominados, por su parte, las tres principales prácticas son la resistencia, la apropiación y la innovación (Bonfil, 1991).

Ambas herramientas teóricas fueron de mucha utilidad para el desarrollo de la investigación por cuanto contribuyeron a procesar el fenómeno de la institucionalización de la educación indígena en el Ecuador como un proceso, finalmente, de disputa entre el Estado y las comunidades indígenas por la definición y el control de la educación implementada en las zonas rurales; así como a interrogarse por las dimensiones que fueron objeto de dicha disputa y las estrategias puestas en juego tanto por el Estado como por las comunidades.

En términos metodológicos, la investigación optó por realizar el estudio de caso de una experiencia de educación indígena emblemática de los Andes ecuatorianos. Nos referimos al Sistema de Escuelas Indígenas de Cotopaxi, iniciativa que nació en 1975, en los páramos occidentales de la Provincia de Cotopaxi, gracias al trabajo y organización de los líderes indígenas de la zona, conjuntamente con el equipo pastoral de Zumbahua. Una iniciativa que para 1989, año en el que pasa a formar parte del Estado, llegó a estar conformada por un total de veintiún escuelas indígenas, además de varias guarderías y centros de alfabetización; distribuidas en seis de los siete cantones de la provincia (SEIC, 1989).

Para la recolección de información se trabajó a partir de la historia oral (Ripamonti, 2015), no solo por la escasez de fuentes escritas relacionadas con la problemática de investigación, sino también por la necesidad de recuperar la voz de los actores que vivieron y participaron en el proceso de institucionalización de la experiencia arriba señalada, tanto desde el lugar del Estado como desde las escuelas y las comunidades. En esta línea, se realizaron entrevistas a profundidad a los diferentes directores de la Dirección Provincial de Educación Intercultural Bilingüe de Cotopaxi (DIPEIB-C), a varios miembros del equipo de gestión del SEIC, a un total de diecisiete educadores comunitarios, y al presidente del Movimiento Indígena de Cotopaxi.

El trabajo de recolección de información se completó con la revisión de fuentes primarias y secundarias que reposan en los archivos del SEIC, la Inspectoría Salesiana, la DIPEIB-C, y la biblioteca Abya Yala de la Universidad Politécnica Salesiana.

\section{Análisis y resultados}

Para comenzar, es necesario hacer referencia al Sistema de Escuelas Indígenas de Cotopaxi en su primer momento de vida, y, de manera especial, a su forma de organización y propuesta educativa, 
pues solo así se podrá comprender los cambios que dicha iniciativa experimentó el momento en que pasó a formar parte del Estado.

\section{El SEIC, un proyecto educativo comunitario}

El SEIC es una iniciativa de educación que nació en 1975, en los páramos occidentales de la Provincia de Cotopaxi, en lo que actualmente constituyen las parroquias de Zumbahua y Chugchilán. Se trata de una iniciativa que fue impulsada por las mismas organizaciones indígenas de la zona con el apoyo del equipo pastoral de Zumbahua, y que buscó dar respuesta, de una manera harto creativa, a las demandas de formación del momento.

En la década de los 70, las comunidades de la zona se encontraban empeñadas en la implementación de un interesante proyecto de desarrollo endógeno, cuyos tres principales ejes fueron la recuperación de la tierra, el control de la comercialización de los productos agropecuarios, y el acceso a los espacios de poder local (Guerrero, 1992). En el marco de dicho proyecto, el SEIC pasó a cumplir un papel central en la medida en que contribuyó a desarrollar en la población de la zona las habilidades requeridas por el proyecto, entre las que destacan el aprendizaje de la lectura y escritura ${ }^{1}$, el manejo de las operaciones matemáticas básicas y el desarrollo de un pensamiento propio (Herrán, 2008).

En materia del modelo de gestión, el SEIC fue una iniciativa comunitaria en todo el sentido de la palabra, pues no solo que fue gestada por las comunidades y buscó atender a sus necesidades, sino que -y esto es lo más relevante- fue controlada y direccionada por ellas. Las comunidades eran las que definían el perfil de los educadores y tenían la potestad para seleccionarlos y retirarles del cargo, en caso de que no cumplieran con

El nivel de analfabetismo en la zona en cuestión era alarmante en la década de 1970. En 1974, más del $80 \%$ de la población indígena mayor de ocho años era analfabeta, situación que era aún más crítica en el caso de las mujeres (Martínez \& Burbano, 1994). lo esperado. De igual manera, las comunidades tenían el poder para definir los objetivos del proceso formativo, así como hacer el seguimiento y validar el trabajo de los educadores en el día a día (Analuisa, 2014).

El control que las comunidades tenían sobre el proceso educativo y los educadores, en particular, fue posible gracias al hecho de que eran las mismas comunidades las que sostenían a los educadores, ya sea a través del trabajo de sus tierras o la entrega regular de comida o donaciones en dinero. De esta manera, se aseguraba que los educadores respondieran al mandato de las comunidades y cumplieran con lo acordado en sus asambleas.

Con relación al proyecto educativo, en particular, el SEIC impulsó una propuesta innovadora que se acopló a la realidad de la zona y que se alineó con su proyecto político. En relación al primer punto, dos de los aspectos más novedosos fueron la duración de la formación y su organización. En relación al primer punto, la propuesta tenía una duración de cuatro años con la finalidad de que los estudiantes pudieran concluir el ciclo formativo, pues luego de este tiempo era muy común que migraran a las ciudades aledañas por cuestiones laborales (Martínez \& Burbano, 1994). Con relación al segundo aspecto, el proceso de aprendizaje en el SEIC se organizó por cartillas y no por niveles rígidos, y cada estudiante tenía la capacidad de avanzar a su propio ritmo, de acuerdo con su tiempo y posibilidades (Sigcha, 2014).

Relacionado con la propuesta formativa, en estricto sentido, el SEIC -como adelantamos anteriormente- se orientó a desarrollar las habilidades que el proyecto político del momento demandaba: aprender a leer y escribir, aprender a calcular, y aprender a pensar con cabeza propia. La urgencia de aprender a leer y escribir respondía a la necesidad de los comuneros de no dejarse engañar por parte de los terratenientes y los abogados en los procesos de negociación de las tierras. El aprendizaje de las operaciones matemáticas, en cambio, se orientaba a evitar los abusos perpetrados por la población mestiza de las zonas aledañas en los procesos de comercialización de los productos agropecuarios (Lazos \& Lenz, s/a). 
Por último, el aprender a pensar con cabeza propia ocupaba un lugar central en el proyecto educativo, por cuanto implicaba desarrollar en los comuneros no solo la capacidad para analizar críticamente el contexto local desde parámetros propios, sino también la habilidad para plantear soluciones pertinentes a los problemas que les aquejaba como colectividad (Shigui, 2014).

Para desarrollar los aprendizajes arriba descritos, la propuesta formativa se estructuró en cinco áreas académicas: Matemáticas, Ciencias Naturales, Ciencias Sociales, Español y Kichwa; cada una de las cuales contaba con sus propias cartillas para el aprendizaje (Iñiguez \& Guerrero, 1986). No está por demás decir que el trabajo del día a día se lo hacía en la lengua materna de la zona: el Kichwa, y que su metodología de trabajo estaba totalmente influenciada por la pedagogía freiriana (Poaquiza, 2013).

Visto desde la perspectiva de su modelo de gestión y su propuesta formativa, el SEIC constituye una iniciativa que guarda semejanzas con varias experiencias de educación indígena impulsadas en el país y la región. A nivel local, el SEIC mantiene varios elementos en común con las escuelas clandestinas de Cayambe, impulsadas por Dolores Cacuango, y las escuelas autónomas de Simiatug. En términos regionales, la iniciativa mantiene varias similitudes con las escuelas Zapatistas y el proyecto educativo del Consejo Regional Indígena del Valle del Cauca, en Colombia.

Hasta aquí un breve recuento de lo que fue el SEIC en su primer momento de vida: 19751988. Ahora bien, ¿cuáles fueron los cambios que el programa vivió el momento en que pasó a formar parte del Estado en 1989? En lo que sigue presentamos los tres principales impactos que el proceso de institucionalización tuvo en el proyecto educativo y en el día a día de las escuelas.

\section{El aporte a la sostenibilidad de la iniciativa y su cualificación}

Sin lugar a dudas, el proceso de institucionalización fue central para asegurar la sostenibilidad del SEIC en el tiempo. Gracias a dicho proceso, la iniciativa pudo seguir operando las siguientes dos décadas, hasta el 2009, año en el que inició su proceso de desmantelamiento. La sostenibilidad de la iniciativa fue posible gracias a dos factores: el acceso de los educadores comunitarios a nombramientos, por un lado, y la legalización del proyecto educativo y las titulaciones, por el otro.

En relación al primer punto, en 1995, seis años después de que la iniciativa pasara a formar parte del Estado, un grupo importante de educadores comunitarios accedió a nombramientos, con lo cual, pasaron a ser docentes oficiales del sistema de educación nacional. El acceso a nombramientos fue de una importancia capital para la sostenibilidad del proyecto, pues no solo significó un salario fijo para los educadores, entregado de manera regular, sino también un incremento importante en sus ingresos (Analuisa, 2014).

Con relación al segundo punto, con el proceso de institucionalización el proyecto educativo ganó legalidad, aspecto también de vital importancia para la sostenibilidad por cuanto contribuyó a que los comuneros de la zona continuaran apostando por la iniciativa educativa, pero ahora con la certeza de que el tiempo invertido por sus hijos tenía como resultado titulaciones oficiales que les permitirían, entre otras cosas, continuar con el siguiente nivel de educación (Farfán, 2014).

La legalización del proyecto fue también importante por cuanto contribuyó a dotar de capital simbólico (Bourdieu, 1999) a las escuelas comunitarias. Con el pasar de los años, en varias comunidades el prestigio de las escuelas indígenas terminó siendo mucho mayor que el de las escuelas hispanas, aspecto que provocó un incremento importante de la matrícula en las primeras (Guamán, 2014).

El otro impacto positivo del proceso institucionalización, y que está estrechamente conectado con el punto anterior, es el que tiene que ver con la cualificación del proyecto. Con el proceso de institucionalización se abrió para los educadores comunitarios la posibilidad de acceder a programas de formación de diferente índole, que les permitió no solo poner al día sus conocimien- 
tos en diferentes campos, sino también concluir la formación secundaria, en un primer momento, y cursar estudios del nivel superior, en un segundo momento (Shigui, 2014).

Lo anterior fue posible gracias a la gestión de la DIPEIB-C en la firma de convenios con diferentes instituciones de formación, entre los que destacan las Escuelas de Fe y Alegría, el Instituto Pedagógico Intercultural Bilingüe Quilloac y la Universidad Técnica de Ambato (Hurtado, 2014)

\section{La pérdida del control de la educación}

Hasta aquí hemos revisado los efectos del proceso institucionalización a nivel de la sostenibilidad del proyecto educativo y su cualificación. Pero ¿qué ocurrió a nivel de la estructura organizativa de la experiencia, y, de manera puntual, en la relación escuela-comunidad? En términos generales, podemos decir que el proceso de institucionalización provocó un lento pero irreversible proceso de pérdida del control del proceso educativo por parte de las comunidades indígenas de la zona, los educadores y el equipo de gestión.

Cuando hablamos de pérdida de control del proceso educativo nos referimos a la pérdida del poder que dichos actores tenían para decidir y hacerse cargo de varios aspectos sensibles del proceso educativo, como era el caso de la definición del perfil de los educadores, la selección y el despido de los educadores, su capacitación, el seguimiento de su trabajo en el día a día del aula, la definición de la propuesta curricular, entre otros. Con el pasar de los años, todas esas atribuciones pasaron a manos del Estado, bajo la figura de la DIPEIB-C, en un primer momento, y de las coordinaciones zonales de educación y las direcciones distritales respectivas, en un segundo momento.

En el proceso arriba aludido fue de central importancia la pérdida del poder que las comunidades de la zona tenían sobre los educadores, aspecto que, como se pudo analizar en la primera parte de esta sección, constituyó el corazón de la iniciativa educativa en su primer momento de vida, y lo que permitió asegurar que aquella respondiera a los intereses de las comunidades indígenas de la zona. La pérdida de control comunitario sobre los educadores se explica, en buena parte, por el acceso de estos últimos a nombramientos, pues el momento en que aquellos pasaron a formar parte del magisterio y a percibir un salario regular por parte del Estado, dejaron gradualmente de responder al mandato de las comunidades y pasaron a responder a los requerimientos y exigencias de su nuevo patrono (Guamangate, 2014).

En el proceso de pérdida del control de la educación se pueden distinguir tres momentos: un primer momento en el que las comunidades perdieron el control de los diferentes procedimientos relacionados con la educación ya sea porque pasaron directamente a manos del Estado, como fue el caso de la selección de los educadores y su capacitación, o a manos del equipo de gestión; y que corresponde al primer momento de vida de la DIPEIB-C: 1989-1999.

Un segundo momento en el que el equipo de gestión de la iniciativa recuperó varios de los procedimientos que en el primer momento pasaron a manos del Estado (la selección de los educadores, la capacitación y el seguimiento de los mismos, entre otros), y que corresponde al período del proyecto de las Redes Amigas (20002007), proyecto de descentralización educativa impulsado por el Estado ecuatoriano con financiamiento y asesoría del Banco Interamericano del Desarrollo.

Y, finalmente, un tercer momento, que inició con el desmantelamiento de la jurisdicción de EIB en el Ecuador, a partir del 2008, y en el que el equipo de gestión perdió el control total de todos los procedimientos relacionados con la iniciativa educativa, incluidos los procesos de selección, capacitación, seguimiento y despido de los educadores, y la definición de la propuesta formativa.

Ahora bien, la pérdida de control de la educación no fue un proceso fluido y exento de tensiones, sino un proceso conflictivo que desató diferentes tipos de repuestas por parte de los 
actores vinculados al SEIC. En este sentido, fue importante el esfuerzo del equipo gestor por asegurar que los nuevos educadores seleccionados fueran de las mismas comunidades en las que se encontraban las escuelas y que cumplieran con el perfil priorizado por las comunidades, así como también el trabajo llevado adelante para dar seguimiento a los educadores (Analuisa, 2014).

Fue importante también el esfuerzo realizado por las escuelas para mantener los procesos de capacitación propios, pues aquellos constituían espacios únicos para motivar a los educadores en la línea original del proyecto educativo, además de ponerles al día en diferentes temáticas relacionadas con el ámbito educativo (Pilatasig, 2014).

\section{La estandarización \\ de la propuesta educativa}

El proceso institucionalización significó, también, un proceso gradual e irreversible de desmantelamiento de la propuesta educativa del SEIC. Ciertamente, con el paso del tiempo, la propuesta formativa no solo que fue perdiendo su formato original, sino que fue dejando, por efecto de lo anterior, de adecuarse a la realidad lingüística y cultural de la zona, y de responder a las necesidades y proyectos de las comunidades.

Tanto los procesos de desmantelamiento de la propuesta formativa como de pérdida de su pertinencia fueron de la mano de la pérdida del control de las comunidades y educadores sobre la iniciativa educativa, lo cual es totalmente entendible, si se considera que la pérdida de control de la educación por parte de dichos actores tuvo como correlato central el incremento de poder del Estado para definir la orientación y forma de organización de la propuesta formativa.

En el proceso de desmantelamiento de la propuesta formativa se pueden distinguir dos momentos. Un primer momento, que corre desde 1989 hasta el 2008 (que corresponde al período de vida de la DIPEIB-C), en el que si bien se logró sostener una propuesta formativa que se orientó a responder a las necesidades de la población de la zona en materia de fortalecimiento de la identidad y de creación de capacidades básicas para salir de las condiciones de pobreza latente en la zona, sufrió cambios importantes a nivel de su organización que mermaron su nivel de adecuación. Nos referimos, de manera puntual, al tema de la ampliación de la formación primaria de cuatro a seis años y a la estandarización de la organización del aprendizaje y promoción: se dejó el sistema de cartillas y se pasó al sistema organización del aprendizaje y promoción por niveles y cursos.

$\mathrm{Y}$, un segundo momento, que corre desde el 2008 en adelante, en el que la propuesta formativa que primó durante cerca de veinte años fue desmantelada radicalmente y en el que las escuelas se alinearon, por presión del Ministerio de Educación, con la propuesta formativa de lo que hasta el 2010 constituía la propuesta curricular de la educación hispana, que poco se adecuaba a la realidad de las comunidades indígenas y su dinámica cotidiana.

En el marco de dicho proceso de desmantelamiento, fue importante el hecho de que se dejara de propiciar la enseñanza en Kichwa, pues en varias de las comunidades zona en cuestión el Kichwa continuaba siendo la lengua de uso cotidiano de la población, y más aun de los niños y niñas (Yanchapanta, 2015). También fue relevante la falta de adecuación de los nuevos contenidos a los niveles y contexto. Este aspecto, más el tema del lengua, terminó haciendo que la educación se convirtiera, muchas de la veces, en una práctica incomprensible y carente de sentido para los niños de la zona, pero también para los educadores (Pilatasig, 2014).

Similar a lo ocurrido con el proceso de pérdida de control de la educación, el desmantelamiento de la propuesta no fue vivido de una manera impávida por parte de las escuelas y las comunidades, sino que fue enfrentado a partir de diferentes estrategias. En este sentido, fue importante el trabajo del equipo de gestión y los educadores para proteger la propuesta curricular original del SEIC frente a los embates de la DIPEIB-C para imponer el currículo genérico de 
la $\operatorname{EIB}^{2}$ (Sigcha, 2014), pero también para mantener el Kichwa como la lengua prioritaria del proceso enseñanza-aprendizaje el momento en que desde el Ministerio de Educación se pidió que se trabajara solo en Español (Yanchapanta, 2015).

También fueron importantes aquellas iniciativas -dispersas en la mayor parte de los casospara adaptar el currículo de la educación hispana al contexto local, o para mantener dentro de la propuesta formativa ciertas prácticas consideradas importantes para la zona, como el trabajo en la chacra (Pilatasig, 2014).

\section{Discusión y conclusiones}

¿Qué podemos decir luego de casi tres décadas de institucionalización del SEIC? ¿Cuál fue el impacto que dicho proceso tuvo en la estructura organizativa de la iniciativa y en su propuesta formativa?

En base lo presentado en el apartado anterior, y a la luz de la perspectiva de la teoría del control cultural (Bonfil, 1991), se puede concluir argumentando que la institucionalización de la educación indígena significó, en la zona objeto de estudio, un proceso paulatino de enajenación de la educación por parte del Estado, que fue de la mano de otros dos procesos que se dieron de manera simultánea: la supresión del proyecto educativo original y la imposición de uno nuevo: el proyecto educativo de la educación hispana.

Es cierto que los educadores y comuneros de la zona llevaron adelante innumerables acciones para resistir dichos procesos y mantener el control de ciertos ámbitos sensibles del proceso educativo, aspecto que contribuyó a que la iniciativa mantuviera un nivel de pertinencia importante durante casi dos décadas; pero también es cierto que dichas acciones no lograron, con el tiempo, detener dichos procesos, y menos aún revertirlos.

Para junio de 2104, mes de corte de la investigación, lo que se hacía en las escuelas del SEIC era similar a lo que se hacía en cualquiera de

2 El embate no fue permanente y dependió del director de la DIPEIB-C pero también de las políticas de la DINEIB. las otras escuelas rurales de la zona. Lo único que les diferenciaba era la presencia de algunos educadores que participaron en la iniciativa desde 1975, la memoria de lo que el SEIC fue y significó para la zona en el lapso de sus casi cuarenta años de vida, y el deseo de que vinieran tiempos mejores para la educación indígena, tiempos en los que, como diría uno de los educadores comunitarios más antiguos del SEIC, “(...) la educación volviera a estar contralada desde abajo” (Guamán, 2014).

La discusión anterior nos remite a la problemática de la relación entre el Estado y los pueblos indígenas, y, de manera puntual, a la problemática de la relación entre el Estado y la educación de los pueblos indígenas. La pregunta que se desprende, entonces, es la siguiente: ¿es posible pensar en proyectos de educación indígena que formen parte del Estado y que, a la vez, respondan a las demandas y proyectos políticos de los contextos locales; o, el hecho de pasar a formar parte del Estado necesariamente supone la cooptación y desmantelamiento de dichas iniciativas? En caso de que la primera opción sea la más factible ¿cuáles serían las condiciones básicas para que aquello se concrete? En caso de que la segunda sea la opción más cercana al contexto actual ¿se deberían pensar en propuestas de educación de carácter autónomo, al estilo de la iniciativa de los Zapatistas en México (Baronet, 2012)?

De entrada, podemos decir que no vemos factible que en el Ecuador actual se desarrollen propuestas de educación indígena de carácter autónomo, al estilo de los Zapatistas, no solo porque la historia de la pueblos indígenas en el Ecuador y su relación con el Estado ha sido muy diferente a la situación mexicana, sino también porque una de las demandas centrales del movimiento indígena ecuatoriano, desde su emergencia en la década de los 80 , ha sido el participar en el Estado y, cuando ha sido posible, el pasar a formar parte de su estructura (Martínez C., 2004).

A partir de la experiencia analizada y de otras que han tenido lugar en la región y que mantienen vigencia hasta el día de hoy, consideramos que si es posible pensar en iniciativas de educación 
que formen parte del Estado y que, a la vez, sean sensibles con las características de la población y respondan a sus necesidades y proyectos colectivos. La condición básica de aquello, sin embargo, es que se asegure el control de la educación por parte de las comunidades indígenas.

Tres son, desde nuestra perspectiva, los factores que contribuirían a asegurar un nivel adecuado de control de la educación por parte de las comunidades indígenas. El primero hace alusión al diseño institucional del Estado, y sugiere la necesidad de contar con una instancia especializada de gestión de la educación de la población indígena, controlada por las mismas organizaciones indígenas, y con suficientes recursos y poder para la promoción de aquella ${ }^{3}$.

El segundo factor tiene que ver, más bien, con el modelo de gestión del Estado, y nos remite a un tipo de gestión que más que apostar por emitir directrices desde arriba y controlar que aquellas se concreten localmente, se oriente a crear las condiciones necesarias para que la población indígena defina y actualice sus propuestas educativas y tome a cargo elementos centrales de la gestión de aquellas. Un tipo de gestión que, además, respete las particularidades de cada proyecto educativo y que procese las diferencias existentes entre los proyectos no como un problema, sino como apuestas por adecuarlos a las realidades y dinámicas locales.

El tercer y último factor hace alusión al tema de la organización de las comunidades. La experiencia analizada y también otras de la región demuestran que no puede haber un proceso de apropiación y control de la educación sin comunidades organizadas, pues el control de

3 La experiencia ecuatoriana es importante en este campo, tanto a nivel de sus aciertos como de sus errores, y puede darnos pistas interesantes para pensar el tema. Si algo queda claro en materia de educación indígena luego de varias décadas de ensayos a nivel de la región, es la necesidad de contar con una institucionalidad propia para el desarrollo de aquella, pues la reiterada propuesta de contar con un solo sistema educativo en el que se transversalice el enfoque intercultural, como condición para atender las demandas educativas de la población indígena, no ha surtido efecto en ningún país, incluido el Ecuador de los últimos años. aquella demanda de un proceso permanente de seguimiento y retroalimentación por parte de las comunidades, cuando no de intervención directa para tomar los correctivos necesarios.

Tres factores que nos remiten a una problemática que las organizaciones indígenas del Ecuador y de la región han puesto sobre la mesa de discusión durante las últimas décadas $\mathrm{y}$, de manera especial, durante los últimos años, y que no ha sido procesada con la debida seriedad por la academia ni por los Estados de la región, a saber: la problemática de la plurinacionalidad y sus implicaciones en la organización y gestión de la educación; tema por demás relevante pero que plantea interrogantes para una nueva investigación.

\section{Bibliografía}

Analuisa, M. (19 de junio de 2014). El SEIC y la institucionalización de la EIB. (S. Granda, Entrevistador) Latacunga.

Baronet, B. (2012). Autonomía y educación indígena. Las escuelas zapatistas de la Selva Lacandona de Chiapas. Quito: Abya-Yala.

Bonfil, G. (1991). La teoría del control cultural en el estudio de procesos étnicos. Estudios sobre las culturas contemporáneas, IV (12), 165-204.

Bourdieu, P. (1999). Razones prácticas. Sobre la teoría de la acción. Barcelona: Anagrama.

Farfán, M. (10 de julio de 2014). El SEIC y la institucionalización de la EIB. (S. Granda, Entrevistador) Quito.

Giroux, H. (1995). Teoría y resitencia en educación. México: Siglo veintiuno editores.

Guamán, J. (14 de junio de 2014). El SEIC y la institucionalización de la EIB. (S. Granda, Entrevistador) Comunidad de Saraucsha, Zumbahua.

Guamangate, R. (8 de mayo de 2014). El SEIC y la institucionalización de la EIB. (S. Granda, Entrevistador) Comunidad de Guayama San Pedro, Chugchilán.

Guerrero, P. (1992). El saber del mundo de los cóndores. Identidad e insurgencia de la cultura andina. Quito: Abya-Yala.

Herrán, J. (2008). Testimonio de las escuelas indígenas del Quilotoa. En Movimiento Indígena de 
Cotopaxi, DIPEIB-C e Instituto de Estudios Ecuatorianos, Primera convención internacional de educación intercultural bilingue de Cotopaxi. Memoria. Quito: Instituto de Estudios Ecuatorianos.

Hurtado, A. (23 de mayo de 2014). La DIPEIB-C y el SEIC. (S. Granda, Entrevistador) Pujilí.

Iñiguez, S., \& Guerrero, G. (1986). Un proceso de educación bilingue intercultural en las escuelas indígenas del Quilotoa. Tesis de licenciatura de la Escuela de Filosofía y Pedagogía de la Universidad Politécnica Salesiana.

Lazos, E., \& Lenz, E. (s/a). La educación indígena en el páramo Zumbahueño del Ecuador. Demandas, éxitos y fracaso de una realidad. s/l: s/e.

Martínez, C. (2004). Los misioneros salesianos y el movimiento indígena de Cotopaxi, 1970 2004. Ecuador Debate, 63, 235-268.

Martínez, R., \& Burbano, B. (1994). La educación como identificación cultural y la experiencia de educación indígena en Cotopaxi. Quito: Abya-Yala.

Pérez, M. (2013). Guillermo Bonfil Batalla. Aportaciones al pensamiento social contemporáneo. Cuicuilco, 57, 115-136.

Pilatasig, S. (8 de mayo de 2014). El SEIC y la institucionalización de la EIB. (S. Granda, Entrevistador) Comunidad de Guayama San Pedro, Chugchilán.
Poaquiza, M. C. (2013). Enfoque pedagógico del Sistema de Escuelas Indígenas de Cotopaxi (SEIC) desde la década de 1970 hasta 1998. Tesis presentada para obtener el título de Magister en Gerencia Educativa en la Universidad Andina Simón Bolívar del Ecuador.

Ripamonti, P. (2015). La historia oral o historizar la memoria. En Cátedra Fals Borda de CLACSO, Metodología de la investigación social (págs. 1-18). Argentina: CLACSO.

SEIC (1989). Evaluación participativa de las Escuelas Indígenas de Cotopaxi, 1985 - 1988. Quito: Abya-Yala.

Shigui, R. (10 de abril de 2014). El SEIC y la institucionalización de la EIB. (S. Granda, Entrevistador) Quito.

Sigcha, S. (8 de mayo de 2014). El SEIC y la institucionalización de la EIB. (S. Granda, Entrevistador) Comunidad de Guayama San Pedro, Chugchilán.

Walsh, C. (2013). Pedagogías decoloniales. Práctica insurgentes de resistir, (re)existir y (re)vivir. Quito: Abya-Yala.

Yanchapanta, H. (21 de julio de 2015). El SEIC en el contexto de la Revolución Ciudadana. (S. Granda, Entrevistador) Latacunga. 